\title{
Resonant-tunneling diodes with emitter prewells
}

\author{
Timothy B. Boykin ${ }^{\mathrm{a})}$ \\ Department of Electrical and Computer Engineering, The University of Alabama in Huntsville, \\ Huntsville, Alabama 35899 \\ R. Chris Bowen and Gerhard Klimeck \\ Jet Propulsion Laboratory, California Institute of Technology, MS 168-522, Pasadena, \\ California 91109-8099 \\ Kevin L. Lear \\ Department of Electrical and Computer Engineering, Colorado State University, Fort Collins, \\ Colorado 80523-1373
}

(Received 4 May 1999; accepted for publication 3 July 1999)

\begin{abstract}
Resonant-tunneling diodes (RTDs) incorporating an emitter prewell structure are studied both theoretically and experimentally in order to investigate the utility of the emitter region as a device design parameter. The experiments show a tendency for peak bias, current, and the peak-to-valley ratio to increase for wider prewells, behavior likewise seen in both very simple and detailed calculations. Both the simple and more complete models point to interactions between states associated with the prewell and the main quantum well as the reasons for the increase in peak current. These results suggest design guidelines to affect peak bias, current, or the peak-to-valley ratio of RTDs. () 1999 American Institute of Physics. [S0003-6951(99)01435-7]
\end{abstract}

The quantum well and barriers of a resonant-tunneling diode (RTD) are naturally the most important design parameters available to the device engineer, for they are the primary determinants of the resonance energies and widths, and thus, the peak bias and current. By varying both the materials from which these sections are fabricated and their widths, the designer can achieve a wide range of device characteristics. In terms of design, then, attention is generally lavished upon the quantum well and barriers; in contrast, the emitter region, though the second-most important device structure, is generally only addressed in terms of the doping profile. Although devices incorporating emitter (and collector) prewells have been fabricated and used, ${ }^{1}$ there has been little systematic study of the emitter region as a parameter for affecting peak current and the peak-to-valley ratio (PVR). This is perhaps understandable since under bias there forms an emitter notch, whose quasibound states have long been suspected as the source of much of the valley current. Recent quantitative calculations for high-current-density devices have confirmed that these notch states, broadened by scattering, are in fact responsible for much of the valley current. ${ }^{2}$ Hence, the emitter notch is more often than not regarded as a source of parasitics instead of as a design parameter to be manipulated.

More careful reflection reveals that the emitter notch can do more than act as a source of excess valley current-it can be designed in such a way as to increase the device peak current density. The possibility of using the emitter notch as a design parameter arises directly from the physics of resonant tunneling, for the interaction between the quantum well and emitter notch quasibound states (and even resonances, or virtual states) can broaden the device transmission characteristics, thereby increasing the peak current. That these interactions are quite fundamental can be seen by examining the

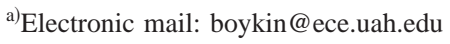

very simple model of Fig. 1, where carriers are incident from the left, over the prewell. In this simple model, all bias is assumed dropped across the barriers and main quantum well (no bias appearing across the prewell), and each region is treated as flatband, offset in energy by its average bias. As shown in Fig. 2, even this simple model displays intriguing resonance behavior, for at certain prewell widths the transmission is markedly higher and broader. This broadening is easily seen to be due to prewell resonances (virtual states) by computing the prewell round-trip phase number $n_{\mathrm{pw}}=2 p / \lambda$ $+\varphi_{B}$, where $p$ is the prewell width, $\lambda$ the carrier wavelength in the prewell, and $\varphi_{B}$ the prewell-barrier interface reflection phase shift, computed for an infinitely thick barrier of the same material as the finite-thickness barrier of the structure. For the 53, 100, and $147 \AA$ prewell structures of Fig. 2, $n_{\mathrm{pw}} \approx 0.995,1.995$, and 2.996 , respectively, giving round-trip phase shifts of almost exactly integral multiples of $2 \pi$ in all cases. Thus, even in very simple models prewell resonances (virtual states) can significantly affect the transmission and we therefore should not be surprised to find that emitter notch quasibound states can have an even greater impact.

The extent to which notch-well interactions affect the current density-voltage $(J-V)$ characteristics can only be quantified through detailed calculations. Here, it is necessary to explicitly integrate over the in-plane wave vector, since

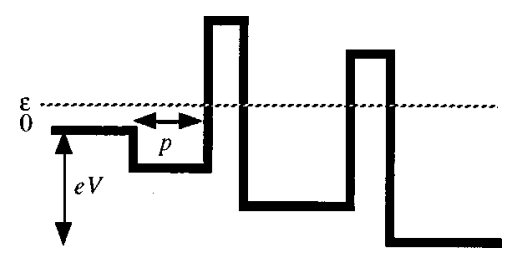

FIG. 1. Band edge of simple model in which transmission is broadened when the round-trip phase shift over the prewell is an integral multiple of $2 \pi$. 


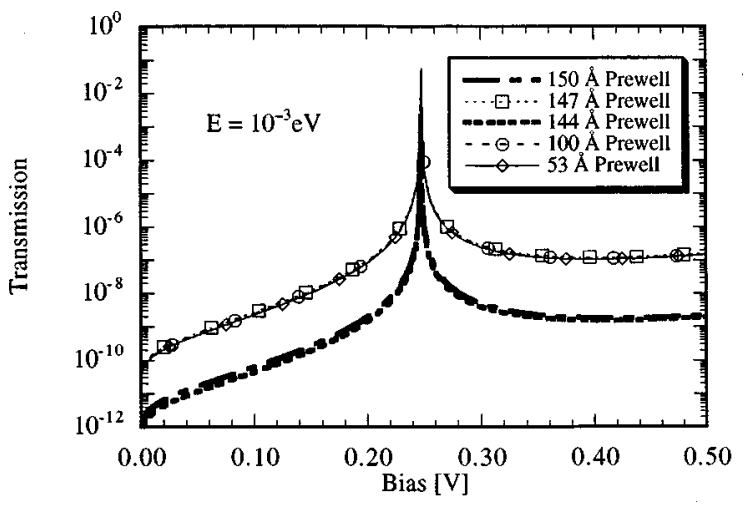

FIG. 2. Transmission vs bias curves for the simple model of Fig. 1. Here, $\epsilon=10^{-3} \mathrm{eV}$, the well width is $45 \AA$, each barrier is $30 \AA$, the barrier height (under zero bias) is $1.0 \mathrm{eV}$, and the prewell is $0.74 \mathrm{eV}$ deep. Effective masses in the bulk and well, prewell, and barriers are $0.067,0.023$, and 0.15 $m_{0}$, where $m_{0}$ is the free-electron mass.

transmission broadening such as that discussed above can lead to unphysical spikes in the $J-V$ characteristics ${ }^{2,3}$ when the Tsu-Esaki ${ }^{4}$ formula is employed. Although a full integration over the in-plane wave vector naturally introduces significant averaging of the well-notch interaction, many effects due to the notch can generally be observed in the $J-V$ curves. Indeed, the magnitude of the virtual-state-related broadening seen above argues strongly that interactions between the main quantum well and both notch quasibound and virtual states can manifest themselves in the $J-V$ characteristics.

This view is further strengthened by the experiments of Lear and co-workers ${ }^{5,6}$ on RTDs differing only in their emitter spacer layers. Each device has collector and emitter doping $N_{D}=2 \times 10^{18} \mathrm{~cm}^{-3}$ (except in the last $1000 \AA$ nearest the emitter spacer, over which the doping was reduced to $10^{17} \mathrm{~cm}^{-3}$ ), a $70 \AA$ undoped emitter spacer, a symmetric, undoped AlAs/GaAs quantum-well structure (45 $\AA$ well and $20 \AA$ barriers), and a $100 \AA$ undoped GaAs collector spacer. The emitter spacer of the control sample was composed entirely of GaAs while the others consisted of an $\mathrm{In}_{0.1} \mathrm{Ga}_{0.9}$ As prewell $(25,45$, or $65 \AA$ wide) adjacent to the emitter barrier, with the balance being GaAs. Room-temperature $I-V$ characteristics for these devices (nominal diameter $5 \mu \mathrm{m}$ ) are shown in Fig. 3, labeled by the width of the $\mathrm{In}_{0.1} \mathrm{Ga}_{0.9} \mathrm{As}$ prewell (the control sample is labeled $0 \AA$ ). Note the marked increase in resonant bias with increasing prewell width, in addition to the increase in resonant current for devices with prewells up to $45 \AA$ wide. The increase in resonant bias with prewell width cannot be explained in terms of zeroth-order electrostatics, for all devices have undoped spacers of identical length, indicating that inelastic scattering is filling quasibound states in the emitter prewell. Likewise, the increase in the PVR for devices with prewells up to $45 \AA$ strongly suggests the presence of prewell/notch-main quantum-well interactions in these devices. To better understand the operation of these RTDs we perform detailed $J-V$ calculations.

The theoretical calculations were performed with NEMO (Refs. 2, 7, and 8) and include full band-structure effects (via the second-near-neighbor, spin-orbit, $s p^{3} s^{*}$ model $^{9}$ ) and integration over the in-plane wave vector along with Hartree self-consistency for determining the potential profile. In the Downloaded 29 Oct 2004 to 129.82.229.173. Redistribution subject

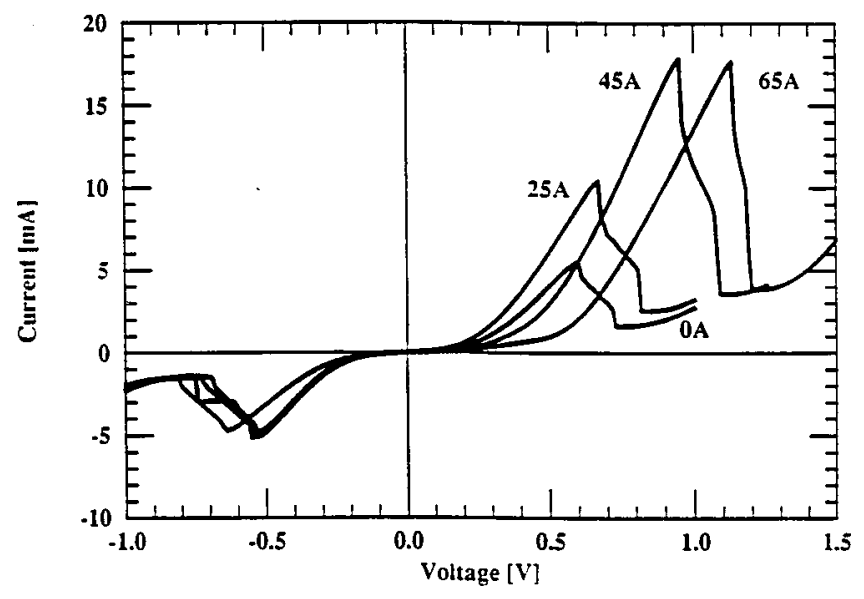

FIG. 3. Experimental $I-V$ characteristics of GaAs/AlAs RTDs with emitter prewells measured by Lear and co-workers (Refs. 5 and 6), labeled by prewell width in $\AA$ (that without prewell is labeled 0).

leads an optical potential $(6.6 \mathrm{meV})$ is used to model broadening of emitter prewell/notch states due to scattering. These calculations model devices like those of Lear and co-workers ${ }^{5,6}$ and series resistance is not included. We have also calculated characteristics for a device with a $35 \AA$ prewell, not represented in the experiments. The calculated results [Fig. 4(a)] nicely match the increase in peak bias with prewell width seen in the experimental curves as well as the rising peak current for larger prewell devices, although they do predict an increased peak current for the $65 \AA$ prewell device over that of the $45 \AA$ device not observed in the measurements. With the exception of the no-prewell device (PVR of 41.2), the calculations also show the tendency evident in the measurements for the PVR to increase with prewell size, the values for the $25,35,45$, and $65 \AA$ devices being 14.5, 22.3, 24.9, and 31.0, respectively. In general, the calculated results display the same trends with respect to prewell size as do the measurements.

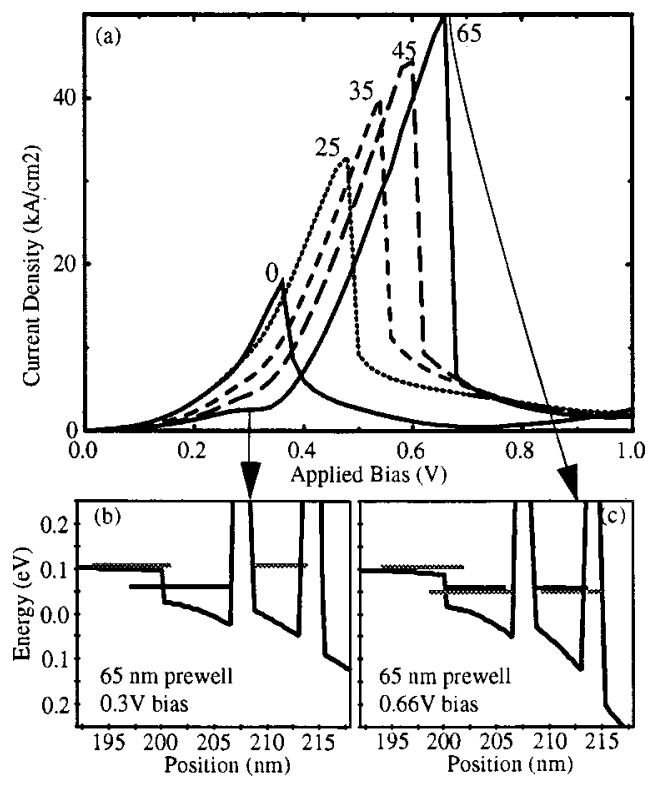

FIG. 4. (a) Calculated $J-V$ characteristics for prewell RTDs similar to those of Fig. 3, labeled by prewell width in $\AA$ (that without prewell is labeled 0 ). (b) Resonance alignments for the $65 \AA$ device at $0.3 \mathrm{~V}$ (virtual and main quantum-well states) and $0.66 \mathrm{~V}$ bias (prewell and main quantum-well

states).
o AIP license or copyright, see http://apl.aip.org/apl/copyright.jsp 
Additional results of the calculations for the $65 \AA$ device [Fig. 4(b)] enable us to explain the $I-V$ characteristics. The current peak occurs when the lowest main quantum-well and emitter prewell quasibound states are aligned, leading to an increase in peak bias with prewell width, the emitter prewell ground state lying lower for wider prewells. The small bump at about $0.3 \mathrm{~V}$ bias results from the interaction of a prewell virtual state (i.e., an above-prewell resonance) and the main quantum-well ground state, showing that virtual states can indeed manifest themselves in properly calculated $J-V$ characteristics. Furthermore, this virtual-state-related feature suggests the reason for the increase of peak current with prewell width. The weakness of the bump compared to the current maximum indicates that more strongly confined prewell states should result in enhanced current peaks, and since lower-lying states in the prewell are better confined than higher, virtual states, higher peak currents should occur for longer prewells, provided that the prewell quasibound states are sufficiently separated.

Although the theoretical and measured characteristics display the same trends, there are some discrepancies between them. First, the experimental peak biases and current densities are consistently higher than the calculated values, the differences in peak bias being larger than can be explained by series resistance alone since for all devices it was only of the order of $1 \Omega$. The most likely explanation is growth-related uncertainties. The doping gradients in the calculations may well be different from those in the actual devices and the actual dopings (in both anode and cathode) could easily differ from the specified values by a factor of 2 . In addition, the actual prewells were likely deeper than specified, the In mole fraction being perhaps as high as 0.15, leading to higher peak biases for the prewell devices. Likewise, it is fairly common to find small differences between the designed and as-grown widths of the barriers and well. Note that here the AlAs barriers are quite thin (7 ML), so that if the actual barriers were even 1-2 ML thinner than specified, the peak currents would be significantly larger. Also, the diameter of each measured device was not exactly determined. Finally, the $65 \AA$ prewell device exhibited some signs of relaxation in the $\operatorname{In}_{0.1} \mathrm{Ga}_{0.9}$ As prewell, which likely accounts for the fact that the measured peak currents of the 45 and $65 \AA$ prewell devices are essentially the same, whereas the calculations predict a higher peak current for the $65 \AA$ prewell device. Despite these differences, it is clear that the calculated and measured characteristics display the same trends, and agree quite well given the uncertainties in growth and fabrication, indicating that prewell/notch-main quantum-well interactions are indeed responsible for the interesting and useful measured $I-V$ characteristics.

We have therefore seen that prewell-main quantum-well interactions are responsible for the enhanced performance of the RTDs fabricated by Lear and co-workers. ${ }^{5,6}$ The calculated results are interesting from a basic physics point of view, since they show that interactions between the main quantum-well and emitter notch states, both quasibound and virtual, can affect the RTD $J-V$ characteristics. Furthermore, and most important for the device designer, both theoretical and experimental results demonstrate that emitter prewells can enhance device performance. The highestperformance devices studied here have well-confined, welldifferentiated prewell quasibound states interacting strongly with the main quantum-well state, so that in designing RTDs device simulations should seek those prewells which enhance this behavior. For our RTDs the results show that both peak current and the PVR tend to increase with prewell size. Taking into account the possibility of relaxation in the $65 \AA$ device, both theory and experiment indicate that in a GaAs/ AlAs RTD with a (nominally) $\operatorname{In}_{0.1} \mathrm{Ga}_{0.9}$ As prewell, a prewell length between 45 and $65 \AA$ gives the best performance. We have, therefore, shown that the emitter notch can be much more than a source of excess valley current: it can be a valuable design parameter.

Two of the authors (T.B.B. and G.K.) thank Joel Schulman for discussions.

${ }^{1}$ R. H. Matthews, J. P. Sage, T. C. L. G. Sollner, S. D. Calawa, C.-L. Chen, L. J. Mahoney, P. A. Maki, and K. M. Molvar, Proc. IEEE 87, 596 (1999).

${ }^{2}$ R. C. Bowen, G. Klimeck, R. K. Lake, W. R. Frensley, and T. Moise, J. Appl. Phys. 81, 3207 (1997).

${ }^{3}$ T. B. Boykin, R. E. Carnahan, and K. P. Martin, Phys. Rev. B 51, 2273 (1995).

${ }^{4}$ R. Tsu and L. Esaki, Appl. Phys. Lett. 22, 562 (1973).

${ }^{5}$ K. L. Lear, W. S. Lee, and J. S. Harris, Jr., Proceedings of the 47th Annual Device Research Conference, June 19-21, 1989, Massachusetts Institute of Technology, Cambridge, Massachusetts.

${ }^{6}$ K. L. Lear, dissertation, Stanford University (1990).

${ }^{7}$ G. Klimeck, R. Lake, R. C. Bowen, W. R. Frensley, and T. Moise, Appl. Phys. Lett. 67, 2539 (1995)

${ }^{8}$ R. Lake, G. Klimeck, R. C. Bowen, and D. Jovanovic, J. Appl. Phys. 81, 7845 (1997)

${ }^{9}$ P. Vogl, H. P. Hjalmarson, and J. D. Dow, J. Phys. Chem. Solids 44, 365 (1983) 